\title{
Deep radio images of the HEGRA and Whipple TeV sources in the Cygnus OB2 region ${ }^{\star}$
}

\author{
J. Martí ${ }^{1}$, J. M. Paredes ${ }^{2}$, C. H. Ishwara Chandra ${ }^{3}$, and V. Bosch-Ramon ${ }^{4}$ \\ ${ }^{1}$ Departamento de Física, Escuela Politécnica Superior, Universidad de Jaén, Las Lagunillas s/n, 23071 Jaén, Spain \\ e-mail: jmarti@ujaen.es \\ 2 Departament d'Astronomia i Meteorologia, Facultat de Física, Universitat de Barcelona, Martí i Franquès, 1, 08028 Barcelona, \\ Spain \\ e-mail: jmparedes@ub.edu \\ 3 National Center for Radio Astrophysics, TIFR, PB No. 3, Ganeshkhind, Pune 7, India \\ e-mail: ishwar@ncra.tifr.res.in \\ 4 Max Planck Institut für Kernphysik, Saupfercheckweg 1, Heidelberg 69117, Germany \\ e-mail: vbosch@mpi-hd.mpg.de
}

Received 24 April 2007 / Accepted 21 May 2007

\begin{abstract}
Context. The modern generation of Cherenkov telescopes has revealed a new population of gamma-ray sources in the Galaxy. Some of them have been identified with previously known X-ray binary systems while other remain without clear counterparts a lower energies. Our initial goal here was reporting on extensive radio observations of the first extended and yet unidentified source, namely TeV J2032+4130. This object was originally detected by the HEGRA telescope in the direction of the Cygnus OB2 region and its nature has been a matter of debate during the latest years. The situation has become more complex with the Whipple and MILAGRO telescopes new $\mathrm{TeV}$ detections in the same field which could be consistent with the historic HEGRA source, although a different origin cannot be ruled out.

Aims. We aim to pursue our radio exploration of the TeV J2032+4130 position that we initiated in a previous paper but taking now into account the latest results from new Whipple and MILAGRO TeV telescopes. The data presented here are an extended follow up of our previous work.

Methods. Our investigation is mostly based on interferometric radio observations with the Giant Metre Wave Radio Telescope (GMRT) close to Pune (India) and the Very Large Array (VLA) in New Mexico (USA). We also conducted near infrared observations with the $3.5 \mathrm{~m}$ telescope and the OMEGA2000 camera at the Centro Astronómico Hispano Alemán (CAHA) in Almería (Spain).

Results. We present deep radio maps centered on the TeV J2032+4130 position at different wavelengths. In particular, our 49 and $20 \mathrm{~cm}$ maps cover a field of view larger than half a degree that fully includes the Whipple position and the peak of MILAGRO emission. Our most important result here is a catalogue of 153 radio sources detected at $49 \mathrm{~cm}$ within the GMRT antennae primary beam with a full width half maximum (FWHM) of 43 arc-minute. Among them, peculiar sources inside the Whipple error ellipse are discussed in detail, including a likely double-double radio galaxy and a one-sided jet source of possible blazar nature. This last object adds another alternative counterpart possibility to be considered for both the HEGRA, Whipple and MILAGRO emission. Moreover, our multi-configuration VLA images reveal the non-thermal extended emission previously reported by us with improved angular resolution. Its non-thermal spectral index is also confirmed thanks to matching beam observations at the 20 and $6 \mathrm{~cm}$ wavelengths.
\end{abstract}

Key words. radio continuum: stars - X-rays: binaries - galaxies: active - gamma rays: observations

\section{Introduction}

The unidentified $\mathrm{TeV}$ gamma-ray source $\mathrm{TeV} \mathrm{J} 2032+4130$ in the Cygnus OB2 region was originally reported by the HEGRA array of imaging Cherenkov telescopes (Aharonian et al. 2002). Together with HESS J1303-631 (Aharonian et al. 2005a), it is one of the two first extended $\mathrm{TeV}$ sources without a clear counterpart at lower energies. In an attempt to explain the nature of these unidentified extedend gamma-ray sources, several $\mathrm{TeV}$ emission models based on hadronic processes instead of leptonic ones have flourished during the past years (e.g. Torres et al. 2004; Bosch-Ramon et al. 2005). However, the only consensus so far achieved is that these mysterious objects are likely

$\star$ Table 3 is only available in electronic form at http://www. aanda.org to be galactic sources based on their Galactic Plane location and extended nature of their $\mathrm{TeV}$ emission.

During the latest years, several authors have undertaken deep observational efforts to try to identify potential counterparts at other wavelengths (Butt et al. 2003, 2006; Mukherjee et al. 2003). No clear success has been achieved so far at both X-ray, infrared and specially at radio wavelengths where interstellar extinction should be minimal. At present, the deepest radio exploration has been reported by Paredes et al. (2007) based on new observations with the Giant Meter Wave Radio Telescope (GMRT) in Pune (India) and new and archive observations with the Very Large Array (VLA) in New Mexico (USA). As a result of this work, an extended diffuse radio emision (likely of nonthermal nature) and a remarkable population of compact radio sources were detected in coincidence with the Center of Gravity 
$(\mathrm{CoG})$ and few arc-minute radius of the HEGRA extended emission. X-ray and near infrared counterparts have been identified for some of these compact radio sources. Their nature ranges from stellar to likely extragalactic sources but none turned out to be an uncommon or peculiar object from which $\mathrm{TeV}$ emission could be suspected.

More recently, new observations conducted with the Whipple Cherenkov telescope (Konopelko et al. 2007) have resulted in the detection of an extended $\mathrm{TeV}$ source displaced several arc-minutes NE from the HEGRA position but yet consistent with it when all sources of error are considered. The contents of the Whipple error box, corresponding to the (significant) excess ellipse, has been studied by Butt et al. (2007) who report on non-thermal extended radio emission consistent with that found by Paredes et al. (2007). A possible supernova remnant (SNR) interpretation is tentatively proposed for it. They also discuss a conspicuous double-lobed jet-source, now within the new Whipple ellipse and previously noted by Butt et al. (2006) and Paredes et al. (2007). But the latest complication to this puzzle has been added by the water Cherenkov telescope MILAGRO as reported by Abdo et al. (2007). The map of the region obtained by the MILAGRO collaboration indicates the presence of a conspicuous $\mathrm{TeV}$ emitter whose position is consistent with both the HEGRA and Whipple sources. However, the association of any of the Whipple and MILAGRO sources with TeV J2032+4130, although possible, is at present unclear and the existence of different TeV sources in the field cannot be ruled out.

In this context, we present additional radio data covering both the HEGRA, Whipple and MILAGRO peak positions. Firstly, the GMRT observations in Paredes et al. (2007, covering the HEGRA position only) are now reported for the whole field of view of the GMRT antennae primary beam. Secondly, this paper is also based on new VLA observations that we combine with the previous VLA archive data for high fidely mapping of extended emission. Our final maps provide the highest sensitivity and angular resolution radio images so far available for the $\mathrm{TeV}$ sources in the Cygnus OB2 region. In particular, the full analysis of GMRT data has resulted in a catalogue of 153 radio sources reliably detected in the field which we consider to be the most important contribution of this work. The availability of such information in the radio will become very useful in a near future when improved Cherenkov and GLAST observations will be able to further decrease the uncertainty of the $\mathrm{TeV}$ position. The true radio counterpart is then likely to emerge among the many radio sources reported in this paper.

\section{GMRT radio observations and results}

The GMRT observations were carried out at $610 \mathrm{MHz}(49 \mathrm{~cm}$ wavelength) on 2005 July 9 and September 1 according to the $\log$ in Table 1 . They were preliminary reported by Paredes et al. (2007), but limited to the HEGRA circle contents. We refer the reader to this reference for details on the GMRT observing, calibration and data processing techniques. Here, we will provide a full account of our GMRT observing runs including all radio sources within the FWHM of the primary beam of individual antennae. The corresponding wide-field map (about 43 arcmin wide) is presented in Fig. 1. Such field of view was conservatively adopted for two reasons mainly. Firstly, it safely accomodates the location uncertainty of both the HEGRA and Whipple sources. Secondly, it avoids known problems in the modelling of the primary beam response beyond the $50 \%$ level (see Appendix 2 in Garn et al. 2007).
Table 1. Log of GMRT observations used in this paper.

\begin{tabular}{|c|c|c|c|c|c|}
\hline $\begin{array}{l}\text { Date and } \\
\text { project } \\
\text { Id. }\end{array}$ & $\begin{array}{c}\lambda \\
(\mathrm{cm})\end{array}$ & $\begin{array}{l}\text { Anten. } \\
\text { used }\end{array}$ & $\begin{array}{c}\text { IF } \\
\#\end{array}$ & $\begin{array}{c}\text { IF } \\
\text { width } \\
\text { (MHz) }\end{array}$ & $\begin{array}{c}\text { Visibility } \\
\#\end{array}$ \\
\hline $\begin{array}{l}\text { 2005 Jul. } 9 \\
\text { (08JMP01) }\end{array}$ & 49 & all & 1 & $\begin{array}{l}13 \text { (LSB) } \\
16 \text { (USB) }\end{array}$ & $\begin{array}{l}340605 \text { (LSB) } \\
340170 \text { (USB) }\end{array}$ \\
\hline $\begin{array}{c}2005 \text { Sep. } 1 \\
\text { (08JMP01) }\end{array}$ & 49 & all & 1 & $\begin{array}{l}13 \text { (LSB) } \\
16 \text { (USB) }\end{array}$ & $\begin{array}{l}276939 \text { (LSB) } \\
276939 \text { (USB) }\end{array}$ \\
\hline
\end{tabular}

Many radio sources are detected in Fig. 1 when closely inspecting the fits file. We used the automated extracting procedure SAD of the AIPS package in order to produce a list of objects with peak flux density higher than about five times the local noise after primary beam correction. The SAD output was inspected visually and all source detections believed not to be real (i.e. deconvolution artifacts near bright sources) were deleted by hand. The resulting list, considered to be very reliable although not complete at lowest flux density levels, contains 153 radio sources. We present it as a catalogue in Table 3 of the online material accompanying this paper. A sample of this table is included here for illustration purposes of our GMRT catalogue. First and second columns provide the $\mathrm{J} 2000.0$ position in right ascension order. The third and fourth columns give the peak $\left(S_{v}^{\text {Peak }}\right)$ and integrated $\left(S_{v}^{\text {Integ }}\right)$ flux densities. Finally the fifth, sixth and seventh columns contain the result of an elliptical Gaussian fit as returned by the same SAD task. This is an apparent angular size, i.e., not a deconvolved one. All errors are given in parentheses.

Very extended sources with $S_{v}^{\text {Integ }} / S_{v}^{\text {Peak }} \gtrsim 6$, such as some double-lobed radiogalaxies, are not included in Table 3 and their detailed study is reserved for future papers. However, we do pay attention below to some of these objects that we consider relevant to mention for our goals of TeV source identification.

\section{VLA radio observations and results}

The VLA observations were carried out during November 2006 at the 20 and $6 \mathrm{~cm}$ wavelengths with the array being in its $C$ configuration. The corresponding log is given in Table 2. For completeness of the VLA data used in this work, Table 2 also contains other archive observation obtained by other authors in the more compact D configuration of the array and used by us. All VLA data were processed following standard procedures within the AIPS package of NRAO. 3C48 was observed as amplitude calibrator at all wavelengths. Phase calibration was achieved by frequent observations of $2052+365$ and $2007+404$ at 20 and $6 \mathrm{~cm}$, respectively. Phase self-calibration worked satisfactorily at $20 \mathrm{~cm}$ while at $6 \mathrm{~cm}$ the brightest sources were not strong enough to enable it.

The purpose of our VLA observations was twofold. Firstly, the VLA C configuration run was conducted with the idea of combining it (in the Fourier plane) with the other D configuration runs available from the archive. A map computed using $\mathrm{C}+\mathrm{D}$ data is expected to provide both improved angular resolution and sensitivity to extended radio emission in order to better image possible radio counterparts to the $\mathrm{TeV}$ source. The result of combining $\mathrm{C}$ and $\mathrm{D}$ configuration data, using the aips task DBCON, is presented in Figs. 2 and 3. These multi-configuration images display a significant amount of detail both in compact and diffuse emission. Primary beam correction has been applied to them limited to a $30 \%$ response of the primary beam for higher fidelity purposes. 


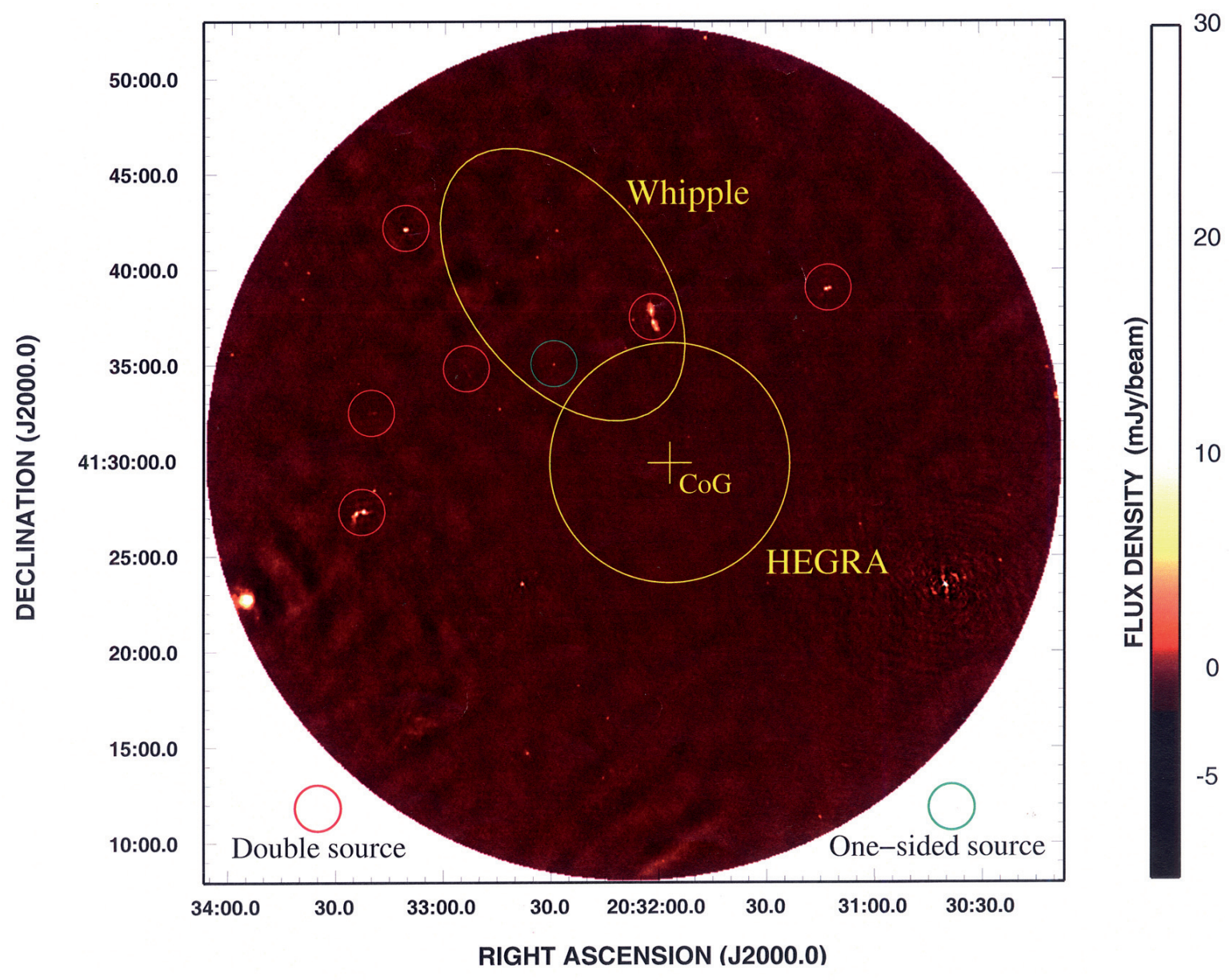

Fig. 1. Wide field radio map of the HEGRA and Whipple TeV sources and its surroundings in the Cygnus OB2 region based on multi-epoch GMRT observations at $49 \mathrm{~cm}$ corrected for primary beam response up to 50\%. The CoG of HEGRA TeV emission and its statistical error are indicated by the central cross, while the central circle ilustrates the $1 \sigma$ radius of its extended angular size. The elongated ellipse approximately represents the Whipple location from Konopelko et al. (2007), believed to correspond to the same TeV source and clearly offset towards the North East. The color scale is logarithmic and shown on the right side. The synthesized beam amounts to 5 .' $^{\prime} 00 \times 4$." $^{\prime \prime}$, with position angle of 33 . $^{\circ} 0$. It is not shown because it would appear just as a small dot at this scale. Resolved double and one-sided jet sources are indicated by color circles red and green, respectively. The rms noise in the central region is about $90 \mu \mathrm{Jy}_{\text {beam }}^{-1}$.

Table 2. Log of VLA observations used in this paper.

\begin{tabular}{cccccc}
\hline \hline $\begin{array}{c}\text { Date and } \\
\text { project } \\
\text { Id. }\end{array}$ & $\begin{array}{c}\lambda \\
(\mathrm{cm})\end{array}$ & $\begin{array}{c}\text { VLA } \\
\text { configuration }\end{array}$ & $\begin{array}{c}\text { IF } \\
\#\end{array}$ & $\begin{array}{c}\text { IF } \\
\text { width } \\
(\mathrm{MHz})\end{array}$ & $\begin{array}{c}\text { Visibility } \\
\#\end{array}$ \\
\hline $\begin{array}{c}\text { 2003 Apr. 29 } \\
\text { (AB 1075) }\end{array}$ & 20 & $\mathrm{D}$ & 2 & 50 & 392125 \\
& 6 & $\mathrm{D}$ & 2 & 50 & 339270 \\
2006 Nov. 21 & 20 & $\mathrm{C}$ & 2 & 50 & 70317 \\
(AM871) & 6 & $\mathrm{C}$ & 2 & 50 & 93624 \\
& & & & & \\
2006 Nov. 28 & 20 & $\mathrm{C}$ & 2 & 50 & 76069 \\
(AM871) & 6 & $\mathrm{C}$ & 2 & 50 & 92433 \\
\hline
\end{tabular}

Secondly, a $\mathrm{C}$ configuration run can also be used to provide true matching beam maps at both 20 and $6 \mathrm{~cm}$. Indeed, observing at $20 \mathrm{~cm}$ in $\mathrm{C}$ configuration provides almost the same synthesized beam as the $6 \mathrm{~cm}$ archive data in D configuration. When these data are appropriately combined, spectral indices can be determined in a more reliable way and specially for extended radio emission. Figure 4 displays the result of combining the matching beam maps quoted above (not shown here) in spectral index mode using the aips task COMB. The field of view covered is larger than a single $6 \mathrm{~cm}$ primary beam because the archive VLA D configuration data consisted of mosaiced pointings at this wavelength.

\section{CAHA near infrared observations and results}

On 2005 April 29, we observed the field of TeV J2032+4130 in the near infrared $K_{\mathrm{s}}$-band $(2.2 \mu \mathrm{m})$ using the Calar Alto $3.5 \mathrm{~m}$ telescope and the OMEGA2000 camera at CAHA. Only five frames could be obtained close in time to the astronomical twilight. Therefore, the sky subtraction is not as perfect as desirable and this limits somewhat the quality of the data. Nevertheless, they are comparable or even better than the 2MASS with a limiting magnitude of $K_{\mathrm{s}}=15.5$. The relevant $K_{\mathrm{s}}$-band fields are displayed as a background gray scale in Figs. 7 and 8. These CAHA observations have been partially published already in Paredes et al. (2007) to whom we refer the reader for further details.

\section{Discussion}

\subsection{The GMRT catalogue of the region}

Possibly the most important contribution of this paper is the GMRT source catalogue compiled in Table 3. Having this 

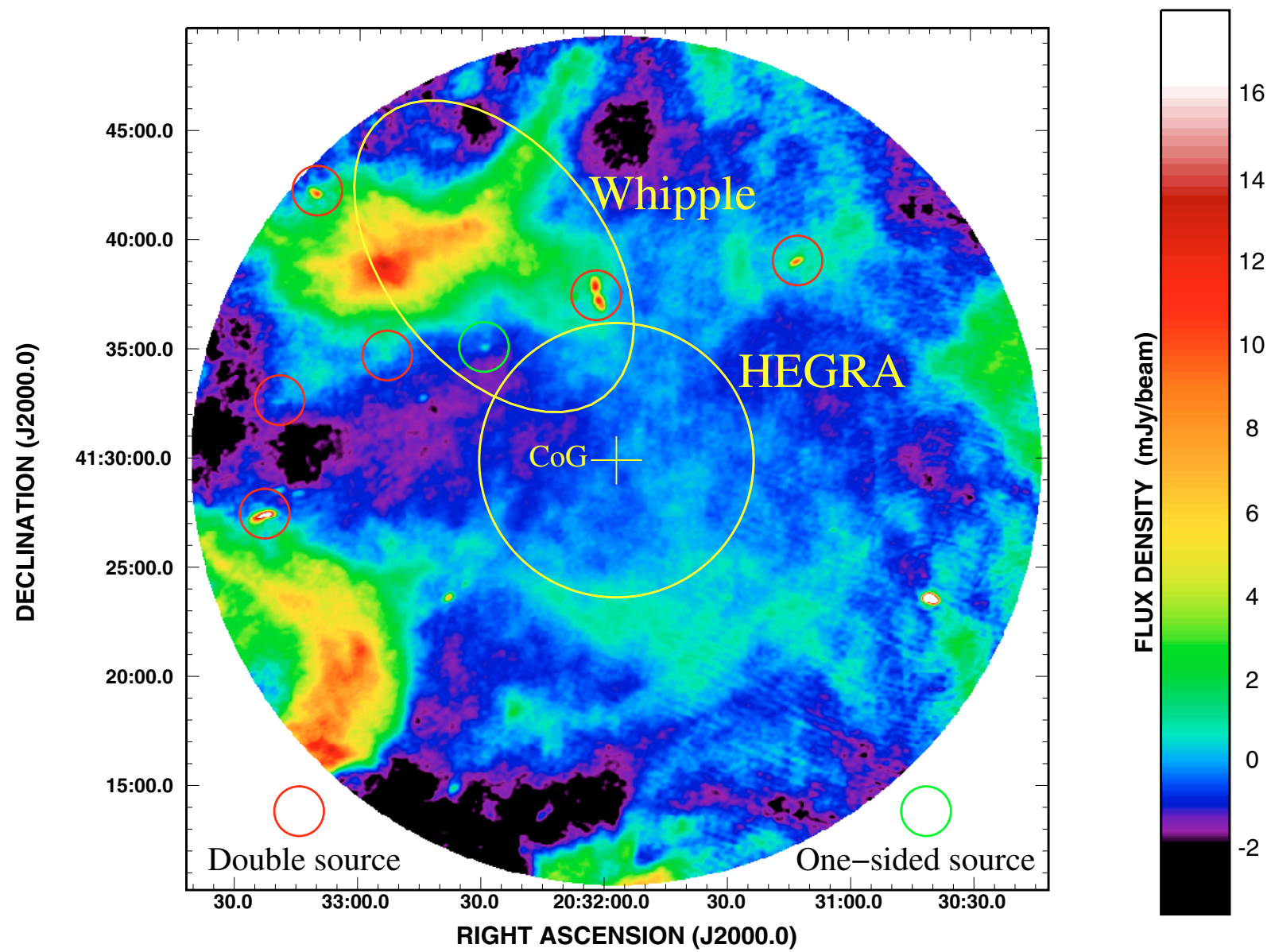

Fig. 2. VLA $20 \mathrm{~cm}$ map of the HEGRA location for the unidentified TeV J2032+4130 source. The location of the HEGRA and Whipple sources are superimposed on it with the same symbols of Fig. 1 . The $20 \mathrm{~cm}$ radio data shown here is a natural weight map made using VLA data taken in $\mathrm{C}$ and $\mathrm{D}$ configuration and combined. The circular map boundary is due to the primary beam correction up to a $30 \%$ response, thus increasing the rms noise toward the edges. The vertical wedge indicates the brightness level in mJy beam ${ }^{-1}$ within limits adapted to better enhance the most

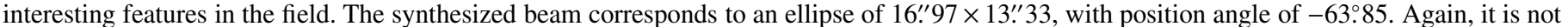
shown because it would appear as a very small dot at this scale. Color circles indicate sources with resolved morphology mainly based on GMRT observations discussed later in the text.

information in hand will likely become a useful reference for radio counterpart identification when future Cherenkov and GLAST observations will be able to narrow the location of the HEGRA, Whipple and MILAGRO sources. The distribution of radio sources with flux density is given by the histogram in Fig. 5. We note here that the number of sources would become significantly higher by $\sim 10^{2}$ if objects at the $3-4 \sigma$ level were accepted in the search.

In Fig. 6 we have plotted the ratio $S_{v}^{\text {Integ }} / S_{v}^{\text {Peak }}$ as a function of peak flux density. The additional dashed lines correspond to the envelope equation

$S_{v}^{\text {Integ }} / S_{v}^{\text {Peak }}=0.85^{-\left(1.2 / S_{v}^{\text {Peak }}\right)}$

and its mirror image with respect to the unity value in the vertical axis. This envelope has been adopted from Bondi et al. (2007) based on their deep source counts using the GMRT at $610 \mathrm{MHz}$. Sources within the two envelope branches are statistically consistent with being compact, or unresolved, while those above can be classified as resolved objects. Out of the 153 objects in Table 3, about $40 \%$ are compact and $60 \%$ appear resolved (61 and 92 radio sources, respectively). The dominance of resolved radio sources is clearly connected the excellent GMRT angular resolution provided by its longest baselines.
The richness of catalogues based on a wide-field instrument such as GMRT allows the prospective of interesting crossidentifications of its $610 \mathrm{MHz}$ radio sources with other wavelength domains. Of course, this task is not limited only to the $\mathrm{TeV}$ sources that originally motivated our work. As an example of quick data mining using the Table 3 catalogue, the object marked with footnote $\left({ }^{d}\right)$ is very likely the lowest frequency detection of the contact binary system Cyg OB2 No. 5 (see e.g. Contreras et al. 1997, for a detailed optical-radio study). Additional hot massive stars worth to be investigated could also be present in Table 3. In particular, sources marked with footnotes $\left({ }^{e}\right)$ and $\left({ }^{f}\right)$ are very close in position to stars Cyg OB2 No. 12 and 9 , respectively.

\subsection{Peculiar radio sources in the HEGRA/Whipple field}

The Whipple ellipse contains some interesting objects outside its overlap with the HEGRA circle but not too far from its edge radius. Their existence has already been noted in the past based on survey data. One of them is the very extended $\left(\sim 10^{\prime}\right)$ radio source $080.45+01.07$ (Furst et al. 1990) previosuly suspected as a possible large scale radio lobe of the nearby microquasar Cygnus X-3 (Martí et al. 2000), but now believed to be a mere HII region of thermal emission nature. The other is the 

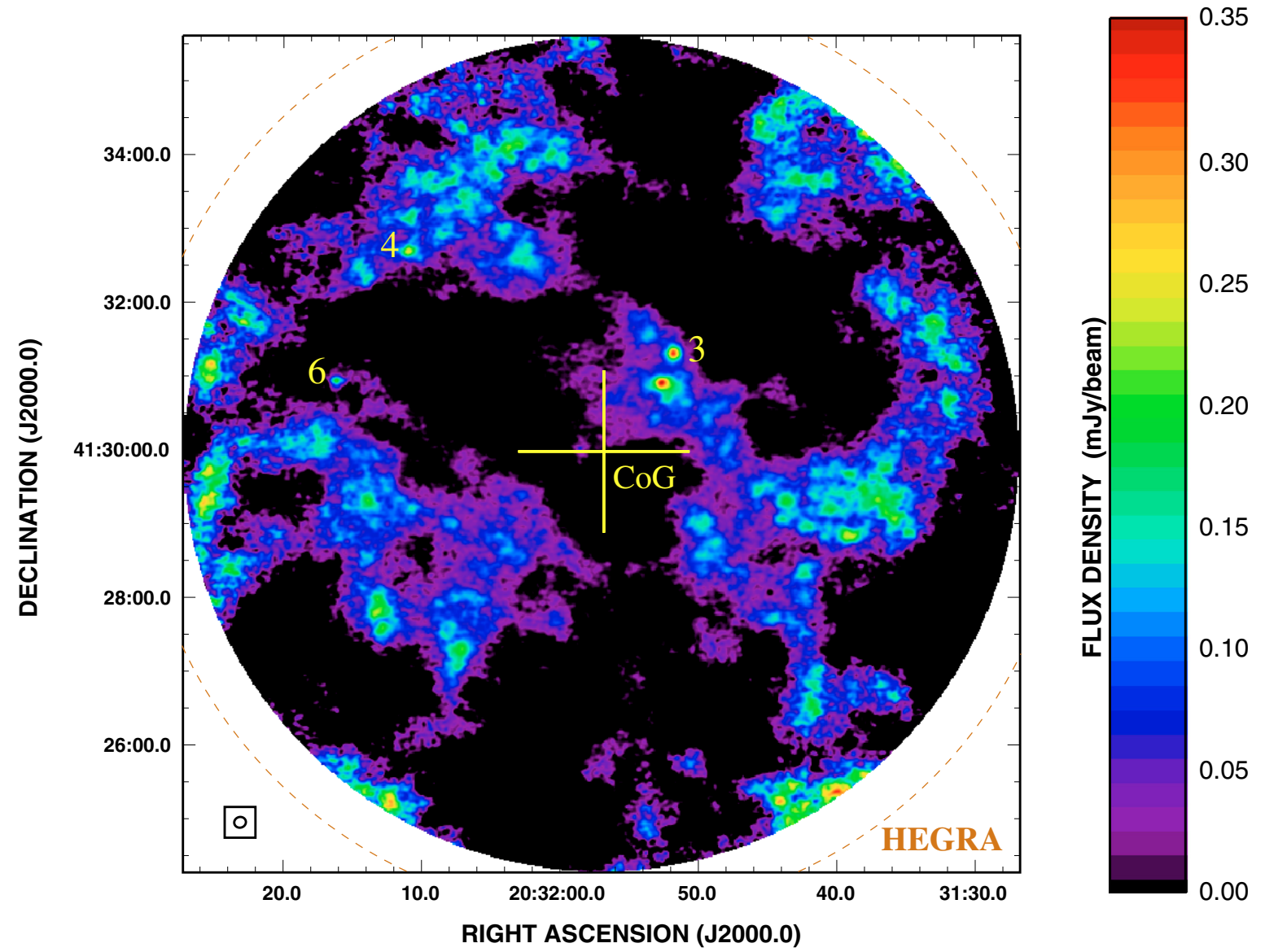

Fig. 3. VLA $6 \mathrm{~cm}$ map of the HEGRA location for the unidentified TeV J2032+4130 source. This is a natural weight map using the C and $\mathrm{D}$ configuration of the array and combined in the $u v$ plane. The HEGRA $1 \sigma$ radius and CoG are the plotted as in previous figure. The vertical

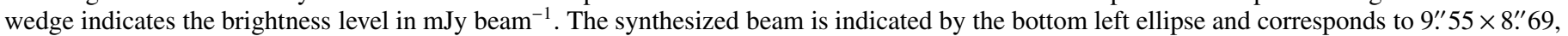
with position angle of $-74^{\circ}$. 44 . Primary beam correction has been also applied limited to a $30 \%$ response. This provides a field of view almost as large as the HEGRA circle. The Id. numbers correspond to GMRT radio sources reported by Paredes et al. (2007) which are also detected here at $6 \mathrm{~cm}$.

prominent double-lobed source mentioned by Butt et al. (2006) and Paredes et al. (2007), also known as NVSS $\mathrm{J} 203201+413722$. None is included in our catalogue due to their very extended nature.

Both objects are imaged here with improved detail. However, the most detailed view of these and other sources in the field comes from the GMRT $49 \mathrm{~cm}$ map in Fig. 1 with about $5^{\prime \prime}$ angular resolution. Thanks to this resolving power, the number of double sources existing in the HEGRA and Whipple field increases significantly to at least six objects (see again Fig. 1). Such abundance reduces the peculiarity of the double-lobed source as a counterpart candidate to be responsible for the TeV J2032+4130/Whipple emission. Moreover, the GMRT map also reveals a third remarkable object, i.e., an unknown onesided jet source also consistent with the Whipple ellipse and not far from the HEGRA circle. We discuss more in detail on these radiosources in the subsections below.

\subsection{NVSS J203201+413722: a likely radio galaxy with an optically-thick compact core}

A detailed GMRT view of this double-lobed source inside the Whipple ellipse is presented in the left panel of Fig. 7. Its morphology is clearly reminiscent of a double-double radio galaxy which has experienced different epochs of jet activity (see e.g.
Schoenmakers et al. 2000). The same field is also shown at $6 \mathrm{~cm}$ from our multi-configuration VLA observations. The primary beam correction for this object at $6 \mathrm{~cm}$ is very high since it is located below the $30 \%$ level response. However, we are confident that its overall $6 \mathrm{~cm}$ morphology is not strongly affected because it is a relatively strong source.

The most interesting issue about NVSS J203201+413722 is revealed by the detection of a compact component in the $6 \mathrm{~cm}$ map located at a $\mathrm{J} 2000.0$ position $20^{\mathrm{h}} 32^{\mathrm{m}} 01.96$ and $+41^{\circ} 37^{\prime} 34^{\prime \prime} 3$ (error $\pm 00^{\prime \prime} 5$ in each coordinate) and with a flux density of $1.1 \pm 0.2 \mathrm{mJy}$. The fact that we do not see it at 20 nor $49 \mathrm{~cm}$, above four times the local $\mathrm{rms}$ noise, implies an optically-thick spectral index of about $\alpha \geq+0.4$. This is possibly due to synchrotron self absorption. Butt et al. (2007) have reported marginal Chandra X-ray detections in the vicinity of NVSS J203201+413722 and their source B is consistent with this optically-thick component. All these facts, together with the central symmetric location of the component with respect to the lobes, strongly suggests that we have identified the true location of the central engine powering the rest of the non-thermal jet source. It is at this precise location where the search for a near infrared counterpart should be conducted in order to test its galactic or, most likely, extragalactic radio galaxy nature. The zoom in the right panel of Fig. 7 also contains in gray scale our CAHA OMEGA2000 observations that reveal no counterpart to the core component brighter than the OMEGA2000 limiting magnitude. 


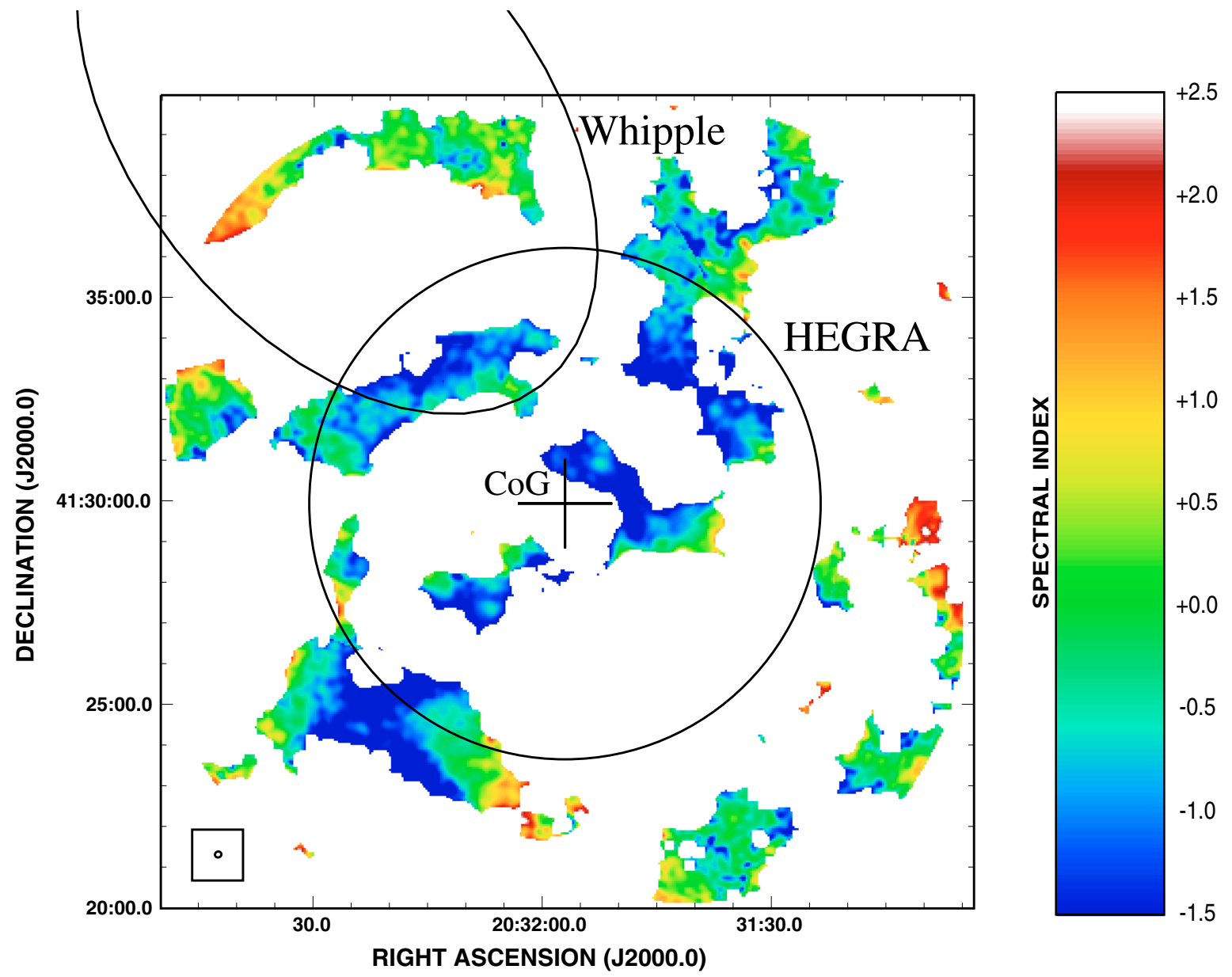

Fig. 4. Spectral index map computed by combining matching beams maps made from VLA $6 \mathrm{~cm}$ data in D configuration and VLA $20 \mathrm{~cm}$ data in $\mathrm{C}$ configuration. The restoring beam used was a circular Gaussian with $15^{\prime \prime}$ diameter at both frequencies shown at the bottom left corner.

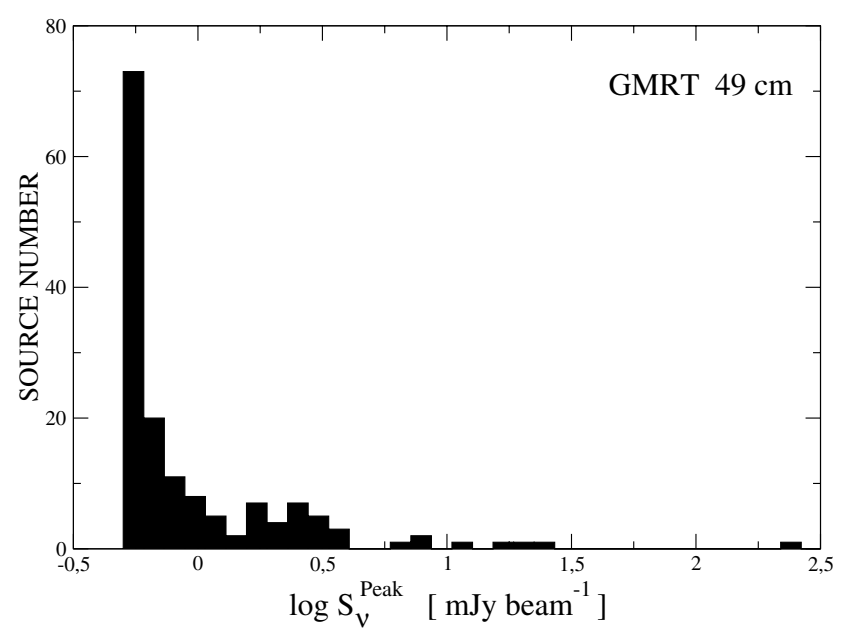

Fig. 5. Histogram showing the distribution of peak flux densities for GMRT radio sources in Table 3 catalogue.

\subsection{A blazar candidate counterpart for the Whipple emission?}

A source with a clearly asymmetric one-sided jet is located inside the Whipple ellipse at $\mathbf{J} 2000.0$ coordinates $20^{\mathrm{h}} 32^{\mathrm{m}} 29.48$ and $+41^{\circ} 35^{\prime} 06^{\prime \prime} 7$, with a peak flux density of $2.8 \mathrm{mJy}$ (see Table 3 for details). This object, included in the GMRT catalogue, is labelled with a green circle in Fig. 1 and a more detailed

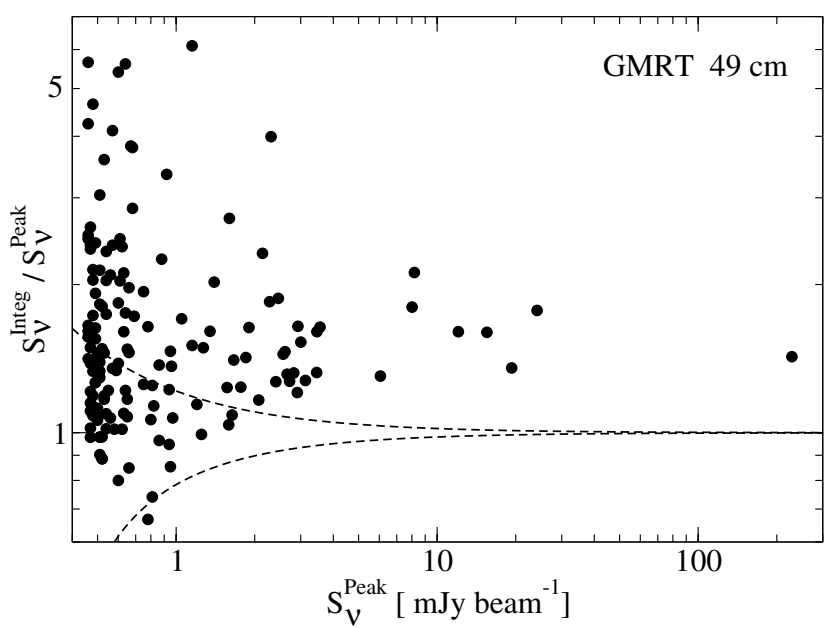

Fig. 6. Ratio of the integrated flux density $S_{v}^{\text {Integ }}$ over the peak flux density $S_{v}^{\text {Peak }}$ for all GMRT radio sources in Table 3 catalogue. Dashed lines are the envelope of loci where sources are statistically consistent with being unresolved (from Bondi et al. 2007).

contour plot is presented in Fig. 8. Jet one-sideness is usually interpreted in terms of strong Doppler boosting of a relativistic jet flowing close to the line of sight. This is the main fingerprint of blazar active galactic nuclei (AGN) which are well known sources of high energy gamma-rays (see e.g. Paredes 2005). 


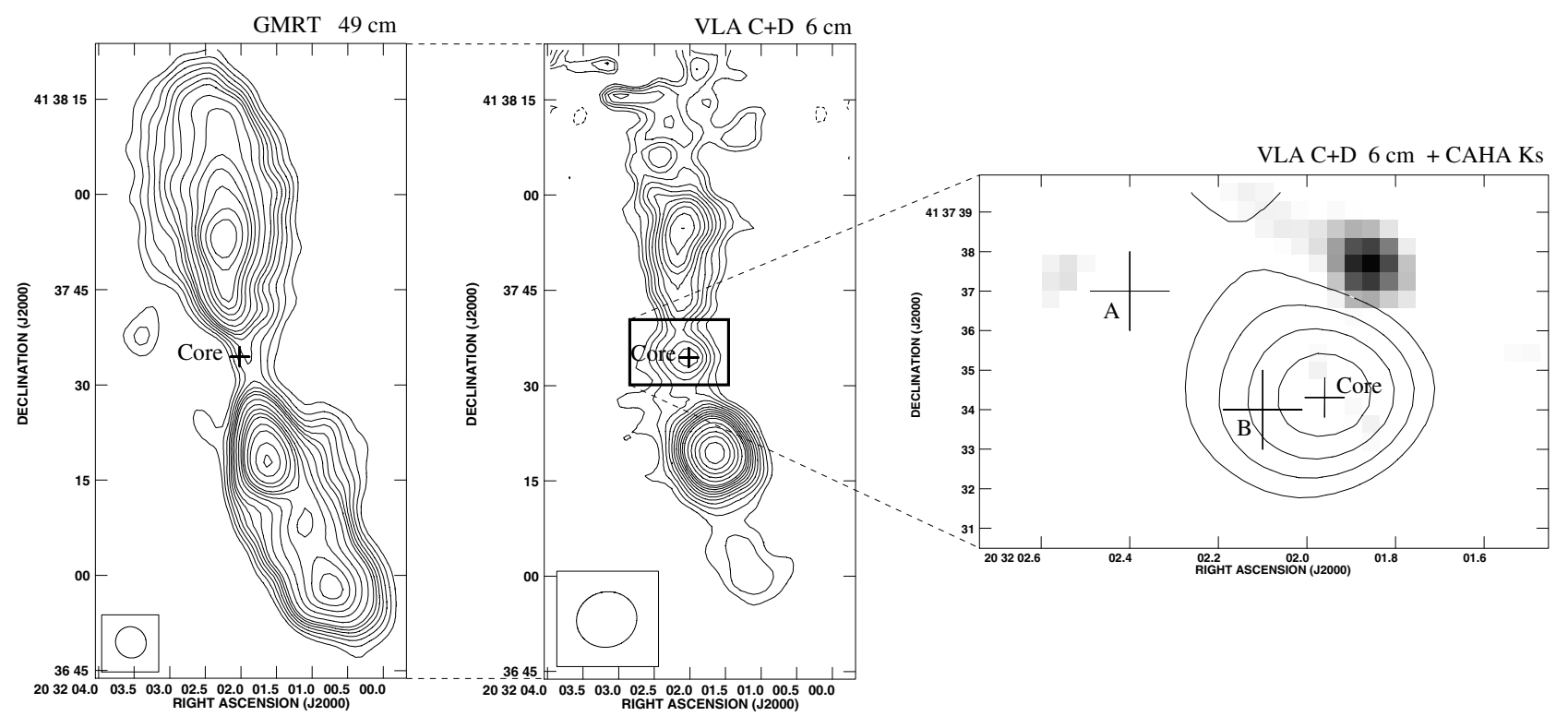

Fig. 7. Left: GMRT radio map of the proposed radiogalaxy at the $49 \mathrm{~cm}$ wavelength computed using a ROBUST value of 0 and excluding baselines shorter than $1.1 \mathrm{k} \lambda$. Primary beam correction has been also applied. Contours correspond to $-4,4,6,8,10,12,14,16,20,25,30,35,40,50$, 60,80 and 100 times $0.12 \mathrm{mJy}_{\text {beam }}{ }^{-1}$, the rms noise. The synthesized beam is shown as an ellipse at the bottom left corner and is equivalent to

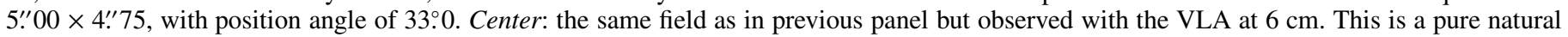
weight, primary beam corrected VLA map produced by combining $u v$ data taken in the D and C configurations of the array. A central thick cross marks the position of a compact component proposed to be the radiogalaxy core. This component is not visible in the left GMRT map also with the same cross symbol for easier comparison. Contours are $-4,4,5,6,7,8,9,10,11,12,13,14,16,18,20,22$ and $_{24}$ times 0.14 mJy beam $^{-1}$. The synthesized beam is similarly shown and corresponds to $9 .{ }^{\prime \prime} 55 \times 8 .^{\prime \prime} 69$, with position angle of -74.44 . Right: zoomed map of the proposed radio core made using the same VLA data of the central panel but using a ROBUST factor of zero for enhanced angular resolution. Contours are

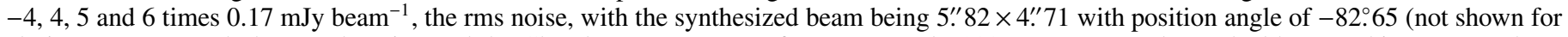
clarity reasons). Both the core location and the Chandra X-ray sources from Butt et al. (2007) are accurately marked here as thin crosses whose size reflects their positional uncertainty. The background gray scale indicates near infrared $K_{\mathrm{s}}$-band emission as observed with the Calar Alto $3.5 \mathrm{~m}$ telescope and the OMEGA2000 camera.

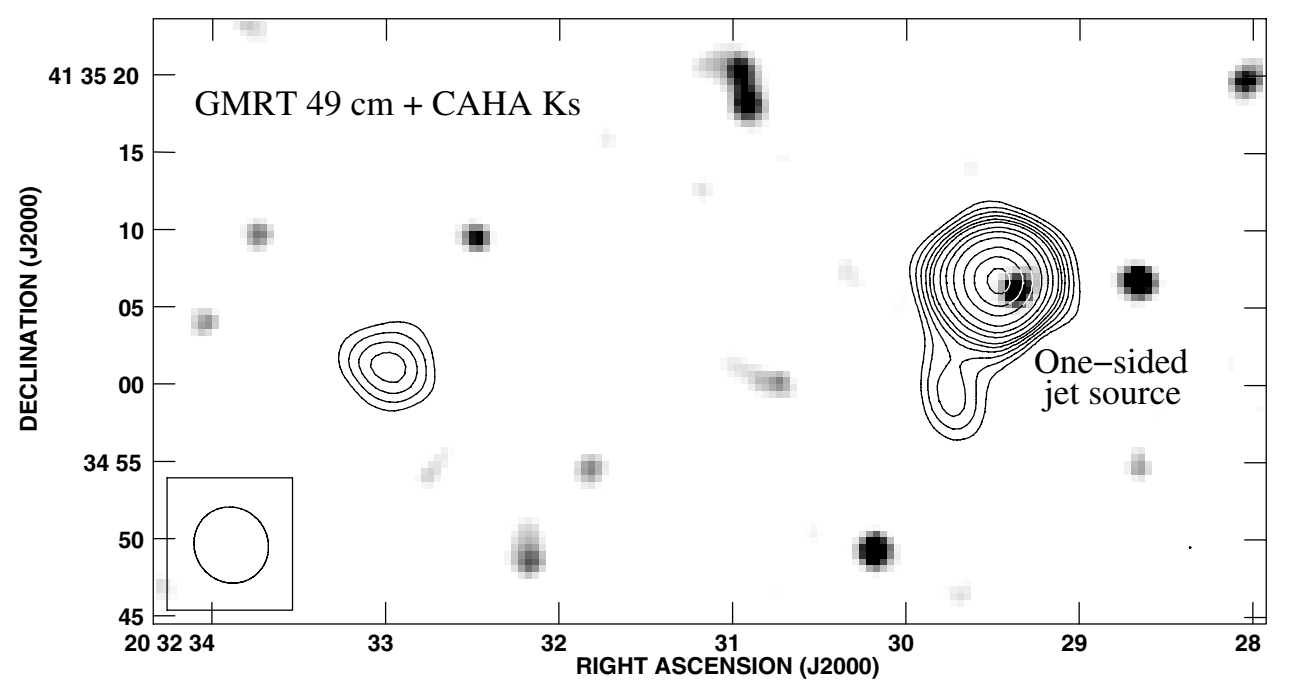

Fig. 8. Contour map of the one-sided jet source detected by the GMRT inside the Whipple ellipse for TeV J2032+4130. Contour levels shown correspond to $-3,3,4,5,6,7,8,10,12,15,20,25$ and 30 times the rms noise of $90 \mu \mathrm{Jy} \mathrm{beam}^{-1}$. This plot is a zoomed region of the same GMRT image as in Fig. 1 and with the same synthesized beam (shown at the bottom left corner). The background gray scale shows the $K_{\mathrm{s}}$-band emission as observed with the Calar Alto $3.5 \mathrm{~m}$ telescope and the OMEGA2000 camera. An obvious stellar-like counterpart candidate is visible in the near infrared for this peculiar radio source.

In this context, one could think of this GMRT one-sided jet source as another potential counterpart candidate for both the HEGRA/Whipple emission deserving further attention in the future. We note here that it could also be consistent with the HEGRA source when taking into account both the statistical and systematic error quoted in Aharonian et al. (2005b). Nevertheless, the extended nature of the HEGRA TeV emission, if confirmed, would be difficult to reconcile with a blazar origin for the radiation and therefore such suggestion has to be considered with caution. 
So far, no entry appears catalogued in the SIMBAD database closer than $20^{\prime \prime}$ from the one-sided jet source position in both radio and X-rays. Using the same Chandra data reported by Butt et al. (2006), we find $\sim 10^{-14} \mathrm{erg} \mathrm{cm}^{-2} \mathrm{~s}^{-1}$ as a resonable X-ray upper limit at its location within the $0.3-10 \mathrm{keV}$ energy range. Such value appears significantly lower than typical X-ray fluxes from other blazar sources, specially when compared with the closest ones (see e.g. Landt et al. 2001), and thus suggesting a high-redshift object unless beaming is not so strong.

Interestingly, the OMEGA2000 image also displayed in Fig. 8 reveals a conspicuous stellar-like object located at $20^{\mathrm{h}} 32^{\mathrm{m}} 29^{\mathrm{s}} 36$ and $+41^{\circ} 35^{\prime} 06^{\prime \prime}$. 1 , i.e., with an offset of $1^{\prime \prime} 5$ between the radio and near infrared position. This value is a few times the combined astrometric uncertainty and roughly one third of the GMRT synthesized beam. Thus, in principle, a radio/near infrared coincidence should not be considered likely. However, an association of the GMRT and CAHA sources cannot be strictly ruled out if the radio peak is not tracing a core but the photocenter of the much brighter arc-second radio jet next to it and below the GMRT angular resolution. From CAHA observations, the magnitude of the near infrared object is $K_{\mathrm{s}}=$ $13.04 \pm 0.02$. It is also present when inspecting the 2 Micron All Sky Survey (2MASS) images with similar brightness in the $K_{\mathrm{s}}$ band. The 2MASS magnitudes for the candidate counterpart to this GMRT source are $J=14.77 \pm 0.03, H=13.40 \pm 0.02$ and $K_{\mathrm{s}}=12.86 \pm 0.03$. Higher angular resolution radio observations, as well as near infrared spectroscopy, is needed to confirm the proposed near infrared counterpart and its suspected blazar or other nature.

\subsection{Extended emission}

The multi-configuration VLA $20 \mathrm{~cm}$ map in Fig. 2 provides a significantly sharper image of the diffuse emission inside the HEGRA circle previously reported in Paredes et al. (2007) with half-arc appearance. The extended emission is broken into multiple patches whose spectral index is predominantly non-thermal as evidenced in the color coding of Fig. 4, thus confirming the Paredes et al. (2007) estimate. Despite of surrounding the $\mathrm{TeV} \mathrm{CoG}$, the morphology of this extended non-thermal feature does not allow an unambiguous diagnosis of its nature and connection with the gamma-ray emission, if any. The same problem occurs when inspecting the multi-configuration $6 \mathrm{~cm}$ image in Fig. 3. At this shorther wavelength, the half-arc becomes more similar to a shell-like feature surrounding the TeV CoG and the two more compact radio sources close to it. A similar conclusion has been reached by Butt et al. (2007) when analyzing the D configuration data alone. While these authors tentatively consider a possible supernova remnant (SNR) reminiscence, this scenario is not clear based on the present data.

\section{Conclusions}

We have conducted a follow-up radio study of the unidentified HEGRA and Whipple TeV sources in the direction of the Cygnus OB2 association. Our main conclusions can be summarized as follows:

1. A catalog of relatively compact radio sources in the field has been reported with a total of 153 objects at the $49 \mathrm{~cm}$ wavelength within the primary beam of GMRT antennae. This catalog, available on-line, is likely to become useful for radio counterpart identication when the $\gamma$-ray positions are improved in the future and for any other objects in the field.
2. Thanks to the excelent angular resolution of GMRT, at least 6 double radio sources are now detected in the field and likely to be double-lobed radio galaxies unrelated to the $\mathrm{TeV}$ emission. The possibility that the prominent double-lobed source, NVSS J203201+413722, previously known close to the HEGRA circle and inside the new Whipple ellipse, is just another unrelated double is therefore enhanced.

3. The combination of GMRT and VLA maps for NVSS J203201+413722 point toward a double-double lobed radio galaxy nature for this object. A compact optically-thick central component has been identified with its central core.

4. An interesting one-sided jet radio source has been discovered inside the position ellipse of the $\mathrm{TeV}$ source detected by Whipple but could also be consistent with the HEGRA source. Its radio morphology is reminiscent of an AGN blazar and it has also a possible stellar-like near infrared counterpart. In the absence of spectroscopic observations, the physical nature of this new GMRT source and its connection with the HEGRA/Whipple emission remain possible but certainly unclear.

5. Finally, the extended radio emission has been also mapped with improved detail with the VLA and accurate spectral index maps produced. A non-thermal shell-like feature around the TeV CoG of TeV J2032+4130 is confirmed, but a physical association with the $\gamma$-ray source cannot be established at present in a clear way.

Acknowledgements. The authors J.M., J.M.P. and V.B.R. acknowledge support by grant AYA2004-07171-C02-01 and AYA2004-07171-C02-02 from the Spanish government, FEDER funds. J.M. is also supported by Plan Andaluz de Investigación of Junta de Andalucía as research group FQM322. V.B.R. gratefully acknowledges support from the Alexander von Humboldt foundation, and thanks the Max-Planck-Institut für Kernphysik for its support and kind hospitality. GMRT is run by the National Centre for Radio Astrophysics of the Tata Institute of Fundamental Research. The NRAO is a facility of the NSF operated under cooperative agreement by Associated Universities, Inc. This paper is also based on observations collected at the Centro Astronómico Hispano Alemán (CAHA) at Calar Alto, operated jointly by the Max-Planck Institut für Astronomie and the Instituto de Astrofísica de Andalucía (CSIC). This research made use of the SIMBAD database, operated at the CDS, Strasbourg, France. This publication makes use of data products from the Two Micron All Sky Survey, which is a joint project of the University of Massachusetts and the Infrared Processing and Analysis Center/California Institute of Technology, funded by the National Aeronautics and Space Administration and the National Science Foundation in the USA.

\section{References}

Aharonian, F., Akhperjanian, A., Beilicke, M., et al. 2002, A\&A, 393, L37 Aharonian, F., Akhperjanian, A. G., Aye, K.-M., et al. 2005c, A\&A, 439, 1013 Aharonian, F., Akhperjanian, A., Beilicke, M., et al. 2005d, A\&A, 431, 197 Abdo, A. A., Allen, B., Berley, D., et al. 2007, ApJ, 658, L33 Bondi, M., Ciliegi, P., Venturi, T., et al. 2007, A\&A, 463, 519 Bosch-Ramon, V., Aharonian, F., \& Paredes, J. M. 2005, A\&A, 432, 609 Butt, Y. M., Benaglia, P., Combi, J. A., et al. 2003, ApJ, 597, 494 Butt, Y. M., Drake, J., Benaglia, P., et al. 2006, ApJ, 643, 238 Butt, Y. M., Jorge, A. C., Jeremy, D., et al. 2007 [arXiv: astro-ph/0611731] Contreras, M. E., Rodríguez, L. F., Tapia, M., et al. 1997, ApJ, 488, L153 Furst, E., Reich, W., Reich, P., \& Reif, K. 1990, A\&ASS, 85, 805 Garn, T., Green, D. A., Hales, S. E. G., et al. 2007, MNRAS, 376, 1251 Konopelko, A., Atkins, R. W., Blaylock, G., et al. 2007, ApJ, 658, 1062 Landt, H., Padovani, P., Perlman, E. S., et al. 2001, MNRAS, 323, 757 Martí, J., Paredes, J. M., \& Peracaula, M. 2000, ApJ, 545, 939

Mukherjee, R., Halpern, J. P., Gotthelf, E. V., Eracleous, M., \& Mirabal, N. 2003, ApJ, 589, 487

Paredes, J. M. 2005, Ap\&SS, 300, 267

Paredes, J. M., Martí, J., Ishwara Chandra, C. H., \& Bosch-Ramon, V. 2007, ApJ, 654, L135

Schoenmakers, A. P., de Bruyn, A. G., Röttgering, H. J. A., et al. 2000, MNRAS, 315,371

Torres, D. F., Domingo-Santamaría, E., \& Romero, G. E. 2004, ApJ, 601, L75 


\section{Online Material}


Table 3. Catalogue of GMRT sources detected at $610 \mathrm{MHz}$.

\begin{tabular}{|c|c|c|c|c|c|c|}
\hline $\begin{array}{c}\alpha_{\mathrm{J} 2000.0} \\
(\mathrm{hms})\end{array}$ & $\begin{array}{c}\delta_{\mathrm{J} 2000.0} \\
(\mathrm{dms})\end{array}$ & $\begin{array}{c}S_{v}^{\text {Peak }} \\
\left(\mathrm{mJy}_{\text {beam }}^{-1}\right)\end{array}$ & $\begin{array}{l}S_{v}^{\text {Integ }} \\
(\mathrm{mJy})\end{array}$ & $\begin{array}{c}a \\
(\operatorname{arcsec})\end{array}$ & $\begin{array}{c}b \\
(\operatorname{arcsec})\end{array}$ & $\begin{array}{c}\text { PA } \\
\text { (degree) }\end{array}$ \\
\hline $203008.779(0.004)$ & $+413316.60(0.03)$ & $8.18(0.11)$ & $17.30(0.32)$ & $8.47(0.11)$ & $5.93(0.08)$ & $83.0(001)$ \\
\hline $203009.090(0.015)$ & $+413326.63(0.21)$ & $2.31(0.10)$ & $9.23(0.51)$ & $11.16(0.51)$ & $8.51(0.39)$ & $4.0(007)$ \\
\hline $203011.448(0.015)$ & $+413452.19(0.15)$ & $2.14(0.11)$ & $4.95(0.34)$ & $8.29(0.42)$ & $6.63(0.33)$ & $68.5(009)$ \\
\hline $203013.065(0.066)$ & +413529.88(0.59) & $0.52(0.11)$ & $0.94(0.29)$ & $9.58(2.02)$ & $4.51(0.95)$ & $54.5(010)$ \\
\hline $203014.295(0.040)$ & $+412454.89(0.52)$ & $0.66(0.11)$ & $1.30(0.30)$ & $7.48(1.24)$ & $6.26(1.03)$ & $18.5(037)$ \\
\hline $203017.861(0.068)$ & $+413012.04(0.64)$ & $0.46(0.11)$ & $0.74(0.27)$ & $8.85(2.11)$ & $4.31(1.03)$ & $126.9(012)$ \\
\hline $203019.401(0.040)$ & $+412423.69(0.58)$ & $0.60(0.11)$ & $1.10(0.29)$ & $7.47(1.36)$ & $5.83(1.06)$ & $1.0(029)$ \\
\hline $203022.154(0.015)$ & $+412810.15(0.14)$ & $1.85(0.11)$ & $2.63(0.25)$ & $6.90(0.42)$ & & $65.8(007)$ \\
\hline $203022.502(0.054)$ & +413921.53(0.38) & $0.64(0$ & $1.12(0.28)$ & & & $98.2(013)$ \\
\hline 203023.0 & +413948 & 0.50 & $0.54(0.21)$ & 6.52( & 3.90 & $178.4(017)$ \\
\hline $203024.657(0.010)$ & +412445.8 & $2.61(0.11)$ & $3.81(0.25)$ & $7.08(0.30)$ & 4.89( & $57.4(005)$ \\
\hline $203028.735(0.070)$ & $+411915.41(0.47)$ & $0.57(0.11)$ & $1.37(0.35)$ & $10.11(1.89)$ & 5.61( & $103.9(012)$ \\
\hline $203029.748(0.032)$ & +411820.76(0.60) & 0.60 & 0.83 & 7.72( & 4.25( & $166.7(012)$ \\
\hline $203034.471(0.036)$ & $+414418.58(0.53)$ & $0.63(0.11)$ & $1.01(0.26)$ & $7.14(1.25)$ & 5.32( & $8.2(024)$ \\
\hline $203037.252(0.036)$ & $+411828.27(0.73)$ & $0.47(0.11)$ & $0.54(0.22)$ & $7.09(1.72)$ & $3.88(0.94)$ & $178.6(015)$ \\
\hline $203037.708(0$ & $+411815.10(0.58)$ & 0.49 & $0.55(0.21)$ & $6.61(1.55)$ & $4.06(\mathrm{C}$ & $37.3(019)$ \\
\hline $203037.787(0.031)$ & $+413144.99(0.58)$ & $0.52(0.11)$ & $0.46(0.18)$ & $6.32(1.40)$ & $3.35(0.74)$ & $162.3(013)$ \\
\hline $203038.443(0.030)$ & $+413333.53(0.42)$ & $0.78(0.11)$ & $1.28(0.27)$ & & $5.57(0.79)$ & $171.1(025)$ \\
\hline $203039.671(0.044)$ & $+413219.40(0.70)$ & $0.49(0.11)$ & $0.76(0.26)$ & $7.39(1.68)$ & 5.00( & $169.1(023)$ \\
\hline 203040.0 & +412330 & $228.61(0.11)$ & $326.27(0.25)$ & & & \\
\hline 203042.99 & +41411 & 0.51 & $0.68(0.24)$ & & & 143. \\
\hline 20304 & +41264 & & & & & \\
\hline 20304 & +41411 & & & & & 178 \\
\hline 203046. & +411937 & 0.5 & 2) & 1) & & 157. \\
\hline 203048 & +411952 & 0.4 & 2) & & & 35.2 \\
\hline 203049.7 & +413612 & 1.0 & 1.79 & 6.6 & 6.07 & 115.1 \\
\hline 203052.8 & +411508 & 0.60 & 0.48 & 4.71 & 4.0 & 62.7 \\
\hline 203055.8 & +411904. & 0.47 & 0.48 & 5.71 & 4) & 43.1( \\
\hline & +412532 . & 0.63 & 1.33 & 10.34 & 4.9 & \\
\hline 203101.1 & +414746 & & $0.50(0$. & & 3.1 & $30.4(C$ \\
\hline $203101.918(0.026)$ & $+411423.73(0.67)$ & $0.54(0.11)$ & $0.55(0.20)$ & 7.42( & $3.27(0.69)$ & $1.4(009)$ \\
\hline $203103.227(0.038)$ & $+414540.33(0.55)$ & $0.52(0.11)$ & $0.46(0.18)$ & 6.71 & $3.16(0.70)$ & $145.9(011)$ \\
\hline 203103.8 & $+412642.57(0.10)$ & $2.66(0.11)$ & $3.50(0.23)$ & 27) & $4.95(0.21)$ & $55.2(007)$ \\
\hline 203104.1 & $+413650.59(0.43)$ & $0.62(0.11)$ & $0.63(0.20)$ & & & $174.9(036)$ \\
\hline 203107 . & +411405 & 0.95 & 5) & & & $56.2(007)$ \\
\hline 203108.7 & +411407. & $0.86(0$ & & & & $49.6(007)$ \\
\hline 20 & +41302 & & & & & \\
\hline 20 & +41295 & 0.46 & & & & 9) \\
\hline 20 & +41390 & 12.0 & 19.3 & & & 130.4 \\
\hline 203113.2 & +413901. & 15.51 & $24.80(0.27)$ & 6.55 & 5.79( & $105.3(002)$ \\
\hline $203113.360(0.043)$ & $+411706.93(0.68)$ & $0.53(0.11)$ & $0.77(0.25)$ & 8.46( & $4.08(0.85)$ & $29.2(011)$ \\
\hline $203114.174(0.028)$ & $+413842.69(0.3$ & & $1.01(0$. & $6.22(0$ & $4.77(0$ & $35.8(021)$ \\
\hline 2031 15.201(0.007) & $+411123.90(0.10)$ & $3.56(0$. & $5.83(0.27)$ & $8.07(0$ & $4.83(0$ & $30.4(002)$ \\
\hline 2031 15.643(0.023) & $+413419.51(0.28)$ & $0.86(0.11)$ & $0.83(0.19)$ & $5.05(0.67)$ & $4.53(0.60)$ & $24.8(050)$ \\
\hline $203116.882(0.016)$ & $+414931.97(0.35)$ & $0.78(0.11)$ & $0.52(0.15)$ & $5.61(0.82)$ & $2.82(0.41)$ & $1.7(008)$ \\
\hline $203119.038(0.054)$ & $+413442.16(0.55)$ & $0.56(0.11)$ & $1.17(0.32)$ & $7.44(1.44)$ & $6.65(1.29)$ & $90.8(070)$ \\
\hline 2031 21.132(0.021) & +413459.74(0.27) & $0.97(0.11)$ & $1.04(0.21)$ & $5.43(0.64)$ & $4.67(0.55)$ & $175.0(031)$ \\
\hline $203124.954(0.052)$ & $+415024.53(0.78)$ & $0.48(0.11)$ & $0.98(0.31)$ & $8.40(1.89)$ & $5.75(1.29)$ & $159.9(023)$ \\
\hline 2031 25.132(0.037) & +414926.34(0.64) & & $0.50(0.20)$ & & & $154.2(010)$ \\
\hline $203125.968(0.072)$ & $+414618.73(0.80)$ & & & $10.16(2.33)$ & & $45.1(015)$ \\
\hline & & & & & & \\
\hline 203129.78 & +411207.8 & 0.66 & 0.96 & & $3.89(C$ & $32.2(007)$ \\
\hline $203132.243(0.065)$ & $+414734.13(1.11)$ & $.11)$ & $1.55(0.41)$ & 14.14( & $5.10(1.06)$ & $150.7(007)$ \\
\hline $203132.448(0.019)$ & $+414756.61(0.25)$ & 1.40 & 2.83( & & $6.35(0$ & $174.1(018)$ \\
\hline 203136.759 & +411740.3 & 0.80 & $0.85(0.21)$ & 6.55 & $3.87(0.56)$ & $29.2(011)$ \\
\hline $203139.166(0.018)$ & $+411819.15(0.26)$ & $0.95(0.1$ & $0.81(0.18)$ & $5.19(0.62)$ & $3.91(0.47)$ & $169.4(017)$ \\
\hline 44 & +415134.76 & 100 & $1.37(0.2$ & $5.80(0$ & $4.68(0.45)$ & $176.3(018)$ \\
\hline $203147.002(0.003)$ & $+415212.80(0.05)$ & $8.01(0.11)$ & $14.40(0.29)$ & $7.91(0.11)$ & $5.40(0.07)$ & $172.5(001)$ \\
\hline $203151.782(0.020)$ & $+413118.29(0.27)$ & $1.15(0.11)^{a}$ & $1.73(0.26)$ & $6.47(0.62)$ & $5.52(0.53)$ & $1.3(025)$ \\
\hline
\end{tabular}


Table 3. continued.

\begin{tabular}{|c|c|c|c|c|c|c|}
\hline $\begin{array}{c}\alpha_{\mathrm{J} 2000.0} \\
(\mathrm{hms})\end{array}$ & $\begin{array}{c}\delta_{\mathrm{J} 2000.0} \\
(\mathrm{dms})\end{array}$ & $\begin{array}{c}S_{v}^{\text {Peak }} \\
\left(\mathrm{mJy} \mathrm{beam}^{-1}\right)\end{array}$ & $\begin{array}{l}S_{v}^{\text {Integ }} \\
(\mathrm{mJy})\end{array}$ & $\begin{array}{c}a \\
(\operatorname{arcsec})\end{array}$ & $\begin{array}{c}b \\
(\operatorname{arcsec})\end{array}$ & $\begin{array}{c}\text { PA } \\
\text { (degree) }\end{array}$ \\
\hline $203158.528(0.044)$ & $+415225.72(0.73)$ & $0.54(0.11)$ & $1.10(0.31)$ & $8.53(1.72)$ & $5.68(1.15)$ & $176.5(020)$ \\
\hline $203200.121(0.044)$ & $+411647.40(0.64)$ & $0.51(0.11)$ & $0.71(0.24)$ & $7.31(1.61)$ & $4.54(1.00)$ & $28.2(018)$ \\
\hline 2032 01.094(0.041) & $+414959.15(0.48)$ & $0.54(0.11)$ & $0.59(0.21)$ & $5.90(1.25)$ & $4.39(0.93)$ & $140.6(028)$ \\
\hline 2032 03.399(0.053) & +413738.40(0.67) & $0.68(0.10)$ & $2.58(0.49)$ & $10.67(1.65)$ & $8.45(1.31)$ & $148.8(027)$ \\
\hline $203204.062(0.046)$ & $+414637.39(0.64)$ & $0.49(0.11)$ & $0.69(0.25)$ & $6.75(1.55)$ & $5.00(1.15)$ & $21.0(031)$ \\
\hline $203204.938(0.023)$ & $+414235.83(0.30)$ & $0.94(0.11)$ & $1.15(0.22)$ & $6.11(0.73)$ & $4.74(0.57)$ & $156.5(019)$ \\
\hline $203206.183(0.023)$ & +41 $1405.73(0.31)$ & $0.96(0.11)$ & $1.31(0.24)$ & $6.73(0.78)$ & $4.80(0.56)$ & $150.4(014)$ \\
\hline $203207.178(0.008)$ & $+414838.47(0.11)$ & $2.72(0.11)$ & $3.46(0.23)$ & $6.23(0.26)$ & $4.85(0.20)$ & $15.1(007)$ \\
\hline 2032 07.337(0.010) & +4137 26.73(0.09) & $2.93(0.11)$ & $4.82(0.27)$ & $7.01(0.26)$ & $5.56(0.21)$ & $8.0(007)$ \\
\hline $203208.365(0.032)$ & $+411311.72(0.52)$ & $0.65(0.11)$ & $0.96(0.25)$ & $7.40(1.26)$ & & $14.9(015)$ \\
\hline 20320 & +41432 & 1.66( & 4) & & & 12.2( \\
\hline 203209.4 & +413557 & 1.57( & & & & $174.5(051)$ \\
\hline 203209.5 & +413 & 0.5 & & & & \\
\hline 203210.9 & +4132 & 0.52( & & & & \\
\hline $203212^{\prime}$ & +41492 & 1.27 & & & & \\
\hline 203213 & +4 & 0.51 & & & & \\
\hline 203213.2 & 1135 & 0.60 & 6) & 7) & 9) & 24.1 \\
\hline 203213.6 & +411503 & 0.48 & 0.52 & 7. & 3 & 53.9 \\
\hline 203214.291 & +411636.0 & 1.90 & 3.11 & 8.1 & 3) & 152.2 \\
\hline 2032 15.361(0.032) & +4122 19.01(0.46) & 0.64( & 0.78( & 6.30( & $0)$ & $160.2(021)$ \\
\hline $203215.595(0.017)$ & $+411328.27(0.26)$ & $1.35(0.11)$ & $2.17(0.27)$ & $7.89(0.65)$ & $4.84(0.40)$ & $154.6(007)$ \\
\hline $203216.289(0.043)$ & +413055.57(0.48) & $0.57(0.11)^{c}$ & $0.77(0.24)$ & 6.04( & $5.37(1.06)$ & 44.2(069) \\
\hline $203216.509(0.051)$ & $+414945.75(0.64)$ & $0.49(0.11)$ & 0.62( & 7.81( & 3.8 & $140.5(012)$ \\
\hline 203218. & +41085 & 0.47 & 1.11 & 10.24 & 5.52 & $177.1(014)$ \\
\hline 203219.8 & +411157. & 0.48 & 0.83 & & & 22) \\
\hline 203221 & +41342 & 052 & & & & \\
\hline 203221.2 & +4109 14.86(0.69) & 0.6 & 1.24 & & 7) & 0) \\
\hline 203222.48 & $+411817.80(0.13)$ & $2.07(($ & 2.4 & & & 24) \\
\hline 203223.3 & +41491 & 0.47 & 0.7 & & & 12) \\
\hline 203227 . & +414 & 1) & & & & \\
\hline 203228 . & +4 & 1) & & & & \\
\hline 203228 . & +41420 & 7 & & & & \\
\hline 203228 . & +41164 & 1) & 4) & ) & & 37.3 \\
\hline 203229.4 & +41350 & 28 & 3.7 & 5. & 5. & 121.7 \\
\hline $203232.739(0.029)$ & $+414630.51(0.38)$ & $0.75(0.11)$ & 0.94 & 6.13( & 4.89 & 23.1(027) \\
\hline $203232.982(0.040)$ & +413501.41(0.45) & 0.59 & 0.79 & 5.70 & 5.54( & $28.2(267)$ \\
\hline $203234.141(0.007)$ & $+414042.63(0.09)$ & $3.13((\mathrm{C})-\mathrm{r}) \mathrm{r}$ & 4.00( & 5.93( & .11( & $1.2(010)$ \\
\hline 2032 34.286(0.040) & +41 24 12.57(0.39) & 0.75 & 45 & 7.31( & 6.28( & $98.4(C$ \\
\hline $203236.683(0.007)$ & $+411445.84(0.10)$ & 3.46( & 5.55 & 7. & .38 & 170.9 \\
\hline $203237.211(0.056)$ & +41 $1453.48(0.77)$ & $0.67(($ & 2.56( & 13.39 & 6.76 & 145.3(009) \\
\hline 203238.196( & $+412336.67(0.01)$ & 19.32 & 26.15 & 6.15 & 5.23 & $167.9(001)$ \\
\hline 203238.7 & +41231 & & & 8 & 8) & \\
\hline 20 & +41 & 0.46 & 1.14 & & & $129.8(009)$ \\
\hline 240 & +411 & $0.82(0.11)^{e}$ & & & & $168.1(029)$ \\
\hline 243 & +411 & & & & & \\
\hline 203244.6 & +41324 & & & & & \\
\hline 203244.7 & +41122 & 0.5 & 1) & & & 0) \\
\hline 203245.3 & +413922. & 1) & 1) & 5.2 & ) & 12.8 \\
\hline 2032 49.093(0.031) & +4110 52.54(0.34) & 0.66( & 0.56 & $5.58(0.96)$ & $3.59(0$ & $46.4(015)$ \\
\hline $203251.789(0.051)$ & $+411056.85(0.62)$ & $0.51(0.11)$ & $0.66(0.23)$ & $8.12(1.81)$ & $3.81(0.85)$ & $42.4(011)$ \\
\hline $203251.980(0.083)$ & $+414656.44(1.02)$ & $0.46(0.10)$ & $1.95(0.54)$ & $11.04(2.50)$ & $9.11(2.06)$ & $149.2(048)$ \\
\hline $203252.081(0.038)$ & $+414500.20(0.58)$ & 476 & 04660 & $5.60(1.37)$ & $4.13(1$ & $175.1(032)$ \\
\hline $203252.697(0.048)$ & +41 1721. & $0.50(0.11)$ & $0.71(0.25)$ & $8.42(1.87)$ & $4.00(0.89)$ & $33.7(011)$ \\
\hline $203252.968(0.051)$ & +4148 11.71(0.71) & $0.46(0$ & $0.76(0.27)$ & 6.97( & $5.61(1$ & $178.7(045)$ \\
\hline $203253.082(0.038)$ & $+413431.10(0.53)$ & $0.88(0.11)$ & $1.98(0.33)$ & $12.45(1.52)$ & $4.27(0.52)$ & $37.2(004)$ \\
\hline $203253.129(0.060)$ & +41 $1049.45(0.56)$ & $0.50(0.11)$ & $0.72(0.25)$ & $8.23(1.84)$ & $4.20(0.94)$ & $54.3(012)$ \\
\hline $203254.292(0.052)$ & +413502.24(0.58) & $0.62(0.11)$ & $1.48(0.35)$ & $9.48(1.63)$ & $5.93(1.02)$ & $45.9(014)$ \\
\hline $203258.046(0.088)$ & +4120 43.94(1.00) & $0.48(0.10)$ & $2.23(0.58)$ & $11.92(2.60)$ & $9.30(2.03)$ & $44.2(035)$ \\
\hline $203300.408(0.095)$ & $+414059.42(1.13)$ & $0.46(0.10)$ & $2.60(0.68)$ & $11.93(2.67)$ & $11.15(2.49)$ & $10.0(133)$ \\
\hline 2033 04.046(0.070) & $+411405.15(1.07)$ & $0.48(0.11)$ & $1.03(0.32)$ & $13.21(3.00)$ & $3.89(0.89)$ & $34.6(006)$ \\
\hline
\end{tabular}


Table 3. continued.

\begin{tabular}{|c|c|c|c|c|c|c|}
\hline $\begin{array}{c}\alpha_{\mathrm{J} 2000.0} \\
(\mathrm{hms})\end{array}$ & $\begin{array}{l}\delta_{\mathrm{J} 2000.0} \\
(\mathrm{dms})\end{array}$ & $\begin{array}{c}S_{v}^{\text {Peak }} \\
\left(\mathrm{mJy}_{\text {beam }}^{-1}\right)\end{array}$ & $\begin{array}{l}S_{v}^{\text {Integ }} \\
(\mathrm{mJy})\end{array}$ & $\begin{array}{c}a \\
(\operatorname{arcsec})\end{array}$ & $\begin{array}{c}b \\
(\operatorname{arcsec})\end{array}$ & $\begin{array}{c}\text { PA } \\
\text { (degree) }\end{array}$ \\
\hline $203305.432(0.041)$ & $+412600.92(0.41)$ & $0.69(0.11)$ & $1.19(0.28)$ & $6.83(1.09)$ & $5.99(0.95)$ & $97.0(049)$ \\
\hline $203307.879(0.079)$ & +411806.28(0.67) & $0.47(0.11)$ & $1.23(0.37)$ & $9.44(2.15)$ & $6.60(1.50)$ & $107.6(025)$ \\
\hline $203310.760(0.015)$ & $+411505.93(0.21)$ & $1.25(0.11)^{f}$ & $1.24(0.20)$ & $5.42(0.50)$ & $4.35(0.40)$ & $167.6(017)$ \\
\hline $203311.272(0.001)$ & +414209.41(0.01) & $24.17(0.11)$ & $42.79(0.28)$ & $7.17(0.03)$ & $5.86(0.03)$ & $55.2(001)$ \\
\hline $203311.771(0.053)$ & +414822.76(0.91) & $0.49(0.11)$ & $1.19(0.35)$ & $10.26(2.24)$ & $5.62(1.23)$ & $160.1(014)$ \\
\hline $203312.374(0.034)$ & $+414730.61(0.41)$ & $0.63(0.11)$ & $0.69(0.21)$ & $5.46(0.99)$ & $4.76(0.87)$ & $23.3(054)$ \\
\hline $203312.622(0.051)$ & $+411534.80(0.75)$ & $0.49(0.11)$ & $0.80(0.27)$ & $8.85(2.00)$ & $4.38(0.99)$ & $32.8(012)$ \\
\hline $203313.248(0.076)$ & $+411529.37(0.73)$ & $0.57(0.10)$ & $2.34(0.53)$ & $11.82(2.18)$ & $8.26(1.52)$ & $121.5(020)$ \\
\hline $203315.139(0.012)$ & +412821.08(0.09) & $2.41(0.11)$ & $3.06(0.23)$ & $6.62(0.31)$ & $4.56(0.21)$ & $97.7(005)$ \\
\hline $203319.499(0.033)$ & +41 $1522.98(0.63)$ & $0.53(0.11)$ & $0.62(0.22)$ & $6.90(1.48)$ & $4.05(0.87)$ & $2.3(016)$ \\
\hline 2033 19.632(0.004) & +412830.71(0.04) & $6.07(0.11)$ & $7.91(0.23)$ & $6.01(0.11)$ & $5.15(0.10)$ & $118.0(005)$ \\
\hline 2033 19.806(0.055) & +4132 33.97(0.30) & $0.92(0.11)$ & $3.08(0.44)$ & $12.72(1.45)$ & $6.22(0.71)$ & $92.9(006)$ \\
\hline 2033 21.157(0.080) & $+413231.85(0.68)$ & $0.46(0.11)$ & $1.16(0.36)$ & $8.97(2.11)$ & $6.75(1.58)$ & $86.1(033)$ \\
\hline $203322.784(0.045)$ & $+411845.37(0.65)$ & $0.54(0.11)$ & $0.94(0.28)$ & 1.62) & $5.23(1.07)$ & $25.8(019)$ \\
\hline $203324.301(0.014)$ & $+413406.05(0.15)$ & $1.59(0.11)$ & $1.65(0.20)$ & $5.21(0.38)$ & $4.75(0.34)$ & $78.8(031)$ \\
\hline 2033 28.186(0.034) & $+414558.07(0.43)$ & $0.65(0.11)$ & $0.76(0.22)$ & $6.45(1.13)$ & $4.29(0.75)$ & $37.3(017)$ \\
\hline 2033 30.962(0.024) & $+413527.45(0.23)$ & $0.94(0.11)$ & $0.89(0.19)$ & $5.73(0$ & $3.92(0.48)$ & $58.6(013)$ \\
\hline 2033 31.553(0.047) & +412959.72(0.69) & 0.49 & 0.62 & 7.84( & 3.85( & 32. \\
\hline $203333.307(0.046)$ & $+411620.50(0.57)$ & 0.68 & $1.94(0.39)$ & $8.75(1-2)-1$ & $7.72(1$ & 19.1( \\
\hline $203333.370(0.055)$ & $+413552.10(0.58)$ & $0.47(0.11)$ & $0.65(0.24)$ & $6.61(1.59)$ & $5.00(1.20)$ & $51.7(034)$ \\
\hline $203336.133(0.023)$ & $+414254.02(0.31)$ & $0.81(0.11)$ & $0.60(0.16)$ & $5.91(0.83)$ & $2.99(0.42)$ & $35.8(008)$ \\
\hline $203338.345(0.041)$ & +414108.39(0.64) & $0.48(0.11)$ & $0.57(0.22)$ & $6.61(1.55)$ & $4.26(1.00)$ & $20.5(021)$ \\
\hline 2033 38.376(0.010) & $+413954.51(0.09)$ & $3.00(0.11)$ & $4.58(0.26)$ & $7.58(0.28)$ & $4.77(0.18)$ & $60.1(003)$ \\
\hline 2033 39.878(0.010) & +413830.08(0.10) & $2.57(0.11)$ & $3.71(0.25)$ & $6.31(0.27)$ & $5.42(0.23)$ & $87.5(011)$ \\
\hline 2033 40.681(0.033) & +41 $4025.78(0.60)$ & $0.58(0.11)$ & $0.59(0.20)$ & $7.80(1.55)$ & $3.13(0.62)$ & $25.6(008)$ \\
\hline 2033 40.761(0.069) & +412702.77(0.81) & $0.64(0.10)$ & $3.59(0.68)$ & $12.02(1.94)$ & $11.07(1.79)$ & $34.7(079)$ \\
\hline $203341.453(0.049)$ & +4126 30.60(0.61) & $0.61(0.11)$ & $1.51(0.36)$ & $8.54(1.50)$ & $6.93(1.22)$ & $30.3(034)$ \\
\hline $203344.630(0.037)$ & $+414022.77(0.52)$ & $1.15(0.10)$ & 7.02(0.73) & 14.01(1.26) & $10.34(0.93)$ & $22.3(012)$ \\
\hline $203350.746(0.032)$ & +412916.47(0.52) & $0.65(0.11)$ & $0.70(0.21)$ & $7.59(1.34)$ & $3.40(0.60)$ & $29.0(008)$ \\
\hline $203352.064(0.020)$ & $+412150.48(0.23)$ & $1.60(0.11)$ & $4.36(0.38)$ & $8.30(0.55)$ & $7.77(0.52)$ & $146.1(040)$ \\
\hline $203359.042(0.055)$ & +4127 41.55(0.66) & $0.51(0.11)$ & $1.09(0.32)$ & & & $151.1(077)$ \\
\hline 2033 59.598(0.063) & +412539.82(0.89) & $0.53(0.11)$ & $1.90(0.47)$ & $11.18(2.21)$ & $7.57(1.49)$ & $26.9(020)$ \\
\hline 2033 & +413536.3 & $2.46(0.11)$ & $4.61(0.29)$ & $7.69(0.34)$ & $5.79(0.26)$ & $91.8(006)$ \\
\hline $203402.483(0.014)$ & $+412454.50(0.12)$ & $2.28(0.11)$ & $4.21(0.29)$ & $7.67(0.37)$ & $5.71(0.27)$ & $105.2(006)$ \\
\hline
\end{tabular}

${ }^{a}$ Same as source \#3, VLA-North and Chandra \#234 within the HEGRA circle in Paredes et al. (2007).

${ }^{b}$ Same as source \#4 within the HEGRA circle in Paredes et al. (2007).

${ }^{c}$ Same as source \#6 within the HEGRA circle in Paredes et al. (2007).

${ }^{d}$ Likely the $610 \mathrm{MHz}$ radio counterpart of the contact binary system Cyg OB2 No. 5.

${ }^{e}$ Likely the $610 \mathrm{MHz}$ radio counterpart of the luminous star Cyg OB2 No. 12.

${ }^{f}$ Likely the $610 \mathrm{MHz}$ radio counterpart of the luminous star Cyg OB2 No. 9. 